\title{
Zero-sum problems with subgroup weights
}

\author{
S. D. Adhikari ${ }^{1}$, A. A. Ambily ${ }^{2} \&$ B. Sury ${ }^{3} *$
}

1 Harish-Chandra Research Institute, Chhatnag Road, Jhunsi, Allahabad 211019, India (email : adhikari@mri.ernet.in)

${ }^{2}$ Statistics \& Mathematics Unit, Indian Statistical Institute, 8th Mile Mysore Road, Bangalore 560059, India (email : ambily@isibang.ac.in)

${ }^{3}$ Statistics \& Mathematics Unit, Indian Statistical Institute, 8th Mile Mysore Road, Bangalore 560059, India (email : sury@isibang.ac.in)

\begin{abstract}
In this note, we generalize some theorems on zero-sums with weights from [1], [4] and [5] in two directions. In particular, we consider $\mathbb{Z}_{p}^{d}$ for a general $d$ and subgroups of $\mathbf{Z}_{p}^{*}$ as weights.

Mathematics Subject Classification 2001: 11B13

Keywords : Cauchy-Davenport theorem, Chevalley-Warning theorem, Zero-

\section{Davenport and Harborth constants for sub- group weights} sum problems

For a finite abelian group $G$ and any non-empty $A \subset \mathbb{Z}$, the Davenport constant of $G$ with weight $A$, denoted by $D_{A}(G)$, is defined (see [2], [3] and [5] for instance) to be the least natural number $k$ such that for any sequence $\left(x_{1}, \ldots, x_{k}\right)$ of $k$ (not necessarily distinct) elements in $G$, there exists a nonempty subsequence $\left(x_{j_{1}}, \ldots, x_{j_{l}}\right)$ and $a_{1}, \ldots, a_{l} \in A$ such that $\sum_{i=1}^{l} a_{i} x_{j_{i}}=0$. Clearly, if $G$ is of order $n$, one may consider $A$ to be a non-empty subset of $\{0,1, \ldots, n-1\}$ and we avoid the trivial case $0 \in A$.

\footnotetext{
*Corresponding author
} 
For natural numbers $n$ and $d$, considering the additive group $G=(\mathbb{Z} / n \mathbb{Z})^{d}$, for a subset $A \subset \mathbb{Z} / n \mathbb{Z} \backslash\{0\}$, we shall use the symbol $D_{A}(n, d)$ to denote $D_{A}(G)$ in this case; for the case $d=1$, the notation $D_{A}(n)$ has been used (see [2], [4], [5], for instance) for $D_{A}(n, 1)$.

Similarly, for $A \subseteq \mathbb{Z} / n \mathbb{Z} \backslash\{0\}$, the constant $f_{A}(n, d)$ is defined (see [1]) to be the smallest positive integer $k$ such that for any sequence $\left(\mathbf{x}_{\mathbf{1}}, \cdots, \mathbf{x}_{\mathbf{k}}\right)$ of $k$ (not necessarily distinct) elements of $(\mathbb{Z} / n \mathbb{Z})^{d}$, there exists a subsequence $\left(\mathbf{x}_{\mathbf{j}_{1}}, \cdots, \mathbf{x}_{\mathbf{j}_{\mathbf{n}}}\right)$ of length $n$ and $a_{1}, \cdots, a_{n} \in A$ such that

$$
\sum_{i=1}^{n} a_{i} \mathbf{x}_{\mathbf{j}_{\mathbf{i}}}=\mathbf{0}
$$

where $\mathbf{0}$ is the zero element of the group $(\mathbb{Z} / n \mathbb{Z})^{d}$. When $d=1$, this was denoted by $E_{A}(n)$ in [2] and [4]. The conjectured relation $E_{A}(n)=D_{A}(n)+$ $n-1$, between the constants $E_{A}(n)$ and $D_{A}(n)$, has been proved by Yuan and Zeng ([14]); and the related general conjecture has also been established by Grynkiewicz, Marchan and Ordaz ([7]) recently.

These constants are respectively the analogues of the Davenport constant (see [10], for instance) and some constant considered by Harborth [8] and others $([6],[9],[12],[13])$. We shall be mainly interested in the numbers $D_{U}(p, d)$ and $f_{U}(p, d)$, where $n=p$, a prime and $U$ a subgroup of $\mathbb{Z}_{p}^{*}$. Here, and henceforth, for a positive integer $n$, we shall write $\mathbb{Z}_{n}$, and $\mathbb{Z}_{n}^{*}$ in place of $\mathbb{Z} / n \mathbb{Z}$, and $\{a \leq n:(a, n)=1\}$ respectively, for simplicity.

We shall often use the following simple observation :

If $U \leq \mathbb{Z}_{p}^{*}$ is a subgroup, then

$$
U=\operatorname{Ker}\left(x \mapsto x^{|U|}\right)=\operatorname{Im}\left(x \mapsto x^{(p-1) /|U|}\right) .
$$

\section{Proposition 1.}

(i) For any subgroup $U \leq \mathbb{Z}_{p}^{*}$, we have

$d\left(D_{U}(p, 1)-1\right)<D_{U}(p, d) \leq \frac{d(p-1)}{|U|}+1$.

Equality holds on the right if $U=\mathbb{Z}_{p}^{*}$, the subgroup $\{1\}$ or the set of quadratic residues.

Also, in general $D_{U}(p, d)=\frac{d(p-1)}{|U|}+1$ if $D_{U}(p, 1)=\frac{p-1}{|U|}+1$. 
(ii) For any subgroup $U \leq \mathbb{Z}_{n}^{*}$, we have $D_{U}(n, d) \geq d(l-1)+1$, where $l$ is the least natural number for which $U$ has a zero-sequence of length l. In particular, if $n=p$, a prime, then $D_{U}(p, d)=\frac{d(p-1)}{|U|}+1$ if $l>\frac{p-1}{|U|}$.

(iii) If $p$ is odd and $U \leq \mathbb{Z}_{p}^{*}$ contains $1,-1$ (in particular, if $\frac{p-1}{|U|}$ is odd), then $D_{U}(p, d) \leq \log _{2}\left(p^{d}+1\right)$.

\section{Proof of (i).}

The inequality $d\left(D_{U}(p, 1)-1\right)<D_{U}(p, d)$ is evident. For the other inequality, write $D=\frac{d(p-1)}{|U|}+1$ for simplicity of notation. Let $\mathbf{a}_{\mathbf{1}}, \cdots, \mathbf{a}_{\mathbf{D}} \in(\mathbb{Z} / p \mathbb{Z})^{d}$ be arbitrary. Write $\mathbf{a}_{\mathbf{i}}=\left(a_{i 1}, \cdots, a_{i d}\right)$ for all $i \leq D$. Consider the $D$ polynomials

$$
\sum_{i=1}^{D} a_{i j} X_{i}^{(p-1) /|U|}, j \leq d .
$$

The sum of the degrees of these homogeneous polynomials is $d(p-1) /|U|$ which is less than $D$. By the Chevalley-Warning theorem, there is a solution $X_{i}=x_{i} \in \mathbb{Z}_{p}$ with not all $x_{i}$ zero. Writing $I=\left\{i: x_{i} \neq 0\right\}$, and $u_{i}=$ $x_{i}^{(p-1) /|U|}$ for $i \in I$, we have $u_{i} \in U$ as observed above. So, we have

$$
\sum_{i \in I} u_{i} \mathbf{a}_{\mathbf{i}}=\mathbf{0} \in(\mathbb{Z} / p \mathbb{Z})^{d}
$$

which means that $D(U, p, d) \leq D=\frac{d(p-1)}{|U|}+1$.

To prove the equalities asserted, use these inequalities and the following zero-sum free sequences. The sequence $(1,0, \cdots, 0),(0,1, \cdots, 0), \cdots,(0,0, \cdots, 1)$ shows $D_{U}(p, d)>d$, when $U=\mathbb{Z}_{p}^{*}$. For the case $U=\{1\}$, we can consider the sequence comprising of each of

$$
(1,0, \cdots, 0),(0,1, \cdots, 0), \cdots,(0, \cdots, 0,1)
$$

repeated $p-1$ times. Finally, if $U$ is the set of quadratic residues, then write $\mathbb{Z}_{p}^{*}=U \sqcup \alpha U$. Then, the sequence of $2 d$ elements

$$
\begin{gathered}
(1,0, \cdots, 0),(-\alpha, 0, \cdots, 0),(0,1, \cdots, 0),(0,-\alpha, 0, \cdots, 0), \\
\cdots \cdots,(0, \cdots, 0,1),(0, \cdots, 0,-\alpha)
\end{gathered}
$$


of $(\mathbb{Z} / p \mathbb{Z})^{d}$ can have no zero-subsequence. Thus, $D_{U}(p, d)>2 d$. This proves (i).

\section{Proof of (ii).}

Consider the sequence of length $\frac{d(p-1)}{|U|}$ in $(\mathbb{Z} / p \mathbb{Z})^{d}$ comprising of each of

$$
(1,0, \cdots, 0),(0,1, \cdots, 0), \cdots,(0, \cdots, 0,1)
$$

repeated $\frac{p-1}{|U|}$ times. If it has a subsequence, say $\mathbf{a}_{\mathbf{1}}, \cdots, \mathbf{a}_{\mathbf{k}}$ and elements $u_{1}, \cdots, u_{k}$ in $U$ such that $\sum_{i=1}^{k} u_{i} \mathbf{a}_{\mathbf{i}}=\mathbf{0} \in(\mathbb{Z} / p \mathbb{Z})^{d}$, then looking at each co-ordinate, we have min $\{l: U$ has a zero-sequence of length $l\} \leq \frac{p-1}{|U|}$, a contradiction of the hypothesis. Thus (ii) is proved.

Proof of (iii).

Note firstly that if $(p-1) /|U|$ is odd, then $1,-1 \in U$ by the observation in the beginning. Write $D=\left\lceil\log _{2}\left(p^{d}+1\right)\right\rceil$ and consider any sequence $a_{1}, \cdots, a_{D}$ of length $D$ in $(\mathbb{Z} / p \mathbb{Z})^{d}$. For each of the $2^{D}-1$ nonempty subsets $J$ of $\{1,2, \cdots, D\}$, look at the sum $\sum_{j \in J} a_{j} \in(\mathbb{Z} / p \mathbb{Z})^{d}$. Note $2^{D}-1 \geq p^{d}$. If these $2^{D}-1$ sums are all distinct elements of $(\mathbb{Z} / p \mathbb{Z})^{d}$, then they must be the various elements of this group and one of them is zero. If these sums are not distinct, there exist two subsets $J_{1} \neq J_{2}$ of $\{1,2, \cdots, D\}$ such that $\sum_{j \in J_{1}} a_{j}=\sum_{i \in J_{2}} a_{i}$. Cancelling off all the terms corresponding to $J_{1} \cap J_{2}$, we have a nonempty subset $J_{0}$ and $\epsilon_{j} \in\{1,-1\}$ such that $\sum_{j \in J_{0}} \epsilon_{j} a_{j}=0 \in$ $(\mathbb{Z} / p \mathbb{Z})^{d}$. This completes the proof.

\section{Remarks.}

(i) If $U \neq \mathbb{Z}_{p}^{*}$ is a subgroup of $\mathbb{Z}_{p}^{*}$ such that $-1 \in U$, then $\{1,-1\}$ is a zero-sum in $U$ of length 2 and hence $\min \{l: U$ has a zero-sequence of length $l\}=2$ and the condition in (ii) of the proposition is not satisfied for the subgroup $U$ of $\mathbb{Z}_{p}^{*}$. For instance, if $p \equiv 1 \quad(\bmod 4)$ and $U$ is the set of quadratic residues mod $p$, then we are in this situation.

(ii) The bound $D_{U}(p, d) \leq \frac{d(p-1)}{|U|}+1$ may not be tight in general. For example, if $U$ is a subgroup of $\mathbb{Z}_{p}^{*}$ of index 3 , for $p=7,13,19$, we have $D_{U}(p, 1)<4$.

(iii) The value of $D_{U}(p, d)$ for the case $U=\{1\}$ is well known. In fact, this case corresponds to the classical Davenport constant and the value is known for all finite abelian $p$-groups (Olson [10]). We shall be using the result in the particular case in the next proposition. 


\section{Proposition 2.}

Let $A=\{1,2, \cdots, r\}$, where $r$ is an integer such that $1<r<p$. We have

(i) $D_{A}(p, d) \leq\left\lceil\frac{d(p-1)+1}{r}\right\rceil$, where for a real number $x$, \lceil\rceil$\rceil$ denotes the smallest integer $\geq x$,

(ii) We have

$$
D_{A}(p, d)>\left[\frac{p}{r}\right] d
$$

Proof of (i).

Write $D=\left\lceil\frac{d(p-1)+1}{r}\right\rceil$. Let $S=\mathbf{a}_{\mathbf{1}}, \cdots, \mathbf{a}_{\mathbf{D}} \in(\mathbb{Z} / p \mathbb{Z})^{d}$ be arbitrary.

Considering the sequence

$$
S^{\prime}=(\overbrace{\mathbf{a}_{\mathbf{1}}, \mathbf{a}_{\mathbf{1}}, \cdots, \mathbf{\mathbf { a } _ { \mathbf { 1 } }}}^{r \text { times }}, \overbrace{\mathbf{a}_{\mathbf{2}}, \mathbf{a}_{\mathbf{2}}, \cdots, \mathbf{a}_{\mathbf{2}}}^{r \text { times }}, \cdots, \overbrace{\mathbf{a}_{\mathbf{D}}, \mathbf{a}_{\mathbf{D}}, \cdots, \mathbf{a}_{\mathbf{D}}}^{r \text { times }}),
$$

obtained from $S$ by repeating each element $r$ times, and observing that the length of this sequence is $\geq d(p-1)+1$ and from Part (i) of Proposition 1, $D_{U}(p, d)=d(p-1)+1$ when $U$ is the subgroup $\{1\}$, the result follows.

Proof of (ii).

Considering the sequence comprising of each of

$$
(1,0, \cdots, 0),(0,1, \cdots, 0), \cdots,(0, \cdots, 0,1)
$$

repeated $\left[\frac{p}{r}\right]$ times, let $\left(t_{1}, t_{2}, \cdots, t_{d}\right)$ be a sum of some of the elements of this sequence with weights $a_{i}$ from the set $A=\{1,2, \cdots, r\}$. If $(0, \cdots, 0,1,0, \cdots, 0)$ with 1 at the $i$-th place is involved in the sum, then we have $0<t_{i} \leq\left[\frac{p}{r}\right] r<$ $p$, and the result follows.

\section{Remarks.}

(i) If $r$ divides $(p-1)$, then from Part (i) we have

$$
D_{A}(p, d) \leq\left\lceil\frac{d(p-1)+1}{r}\right\rceil=\frac{(p-1) d}{r}+1 .
$$

On the other hand, from Part (ii) we have

$$
D_{A}(p, d)>\left[\frac{p}{r}\right] d=\frac{(p-1) d}{r},
$$

thus obtaining the exact value of $D_{A}(p, d)$ in this case. 
(ii) Since the value of the classical Davenport constant is known for all finite abelian $p$-groups (Olson [10]) and for all finite abelian groups of rank 2 (Olson [11]) it is clear that results similar to the above proposition can be obtained for groups of the form $\left(\mathbb{Z} / p^{k} \mathbb{Z}\right)^{d}$ and $(\mathbb{Z} / n \mathbb{Z})^{2}$, for positive integers $k$ and $n$.

The following proposition generalizes some results in [5] and some in [4].

\section{Proposition 3.}

(i) For $U=\mathbb{Z}_{p}^{*}, f_{U}(p, d)=p+d$, if $d<p$.

In particular, $f_{U}(p, p-1)=2 p-1$.

(ii) $f_{U}(p, d) \leq \frac{d(p-1)}{|U|}+p$ if $d<\frac{p|U|}{p-1}$. In particular, $f_{U}(p,|U|) \leq 2 p-1$.

Moreover, if $U$ is the group of quadratic residues, then for $d \leq(p-1) / 2$, we have $f_{U}(p, d)=p+2 d$.

(iii) $f_{U}(p, 1) \geq p-1+D_{U}(p, 1)$ for any subgroup $U$ of $\mathbf{Z}_{p}^{*}$. Further, the equality $f_{U}(p, 1)=p-1+D_{U}(p, 1)$ holds when $D_{U}(p, 1)=1+\frac{p-1}{|U|}$.

Proof of (i).

Let $\mathbf{a}_{\mathbf{1}}, \cdots, \mathbf{a}_{\mathbf{p}+\mathbf{d}} \in(\mathbb{Z} / p \mathbb{Z})^{d}$ be arbitrary. Write $\mathbf{a}_{\mathbf{i}}=\left(a_{i 1}, \cdots, a_{i d}\right)$ for all $i \leq p+d$. Considering the $d+1$ polynomials

$$
\sum_{i=1}^{p+d} a_{i j} X_{i}, j \leq d
$$

and

$$
\sum_{i=1}^{p+d} X_{i}^{p-1}
$$

it follows by the Chevalley-Warning theorem that there is a nontrivial solution $X_{i}=x_{i} \in \mathbb{Z}_{p}$ because the sum of the degrees is $d+p-1<p+d$. If $I=\left\{i: x_{i} \neq 0\right\}$ we have $|I|=p$ because $p+d<2 p$. Therefore,

$$
\sum_{i \in I} x_{i} \mathbf{a}_{\mathbf{i}}=\mathbf{0} \in(\mathbb{Z} / p \mathbb{Z})^{d}
$$


The fact that $f_{U}(p, d)>p+d-1$ follows by considering the following $p$ zerosum-free sequence of length $d+p-1$ :

$$
\underbrace{(0, \cdots, 0), \cdots,(0, \cdots, 0)}_{p-1 \text { times }},(1,0, \cdots, 0),(0,1, \cdots, 0), \cdots,(0,0, \cdots, 1) .
$$

\section{Proof of (ii).}

This has a similar proof. Let $\mathbf{a}_{\mathbf{1}}, \cdots, \mathbf{a}_{\mathbf{2} \mathbf{p}-\mathbf{1}} \in(\mathbb{Z} / p \mathbb{Z})^{d}$ be arbitrary. Let $d<\frac{p|U|}{p-1}$. Write $\mathbf{a}_{\mathbf{i}}=\left(a_{i 1}, \cdots, a_{i d}\right)$ for $i=1,2, \cdots, 2 p-1$. Considering the $d+1$ polynomials

$$
\sum_{i=1}^{t} a_{i j} X_{i}^{(p-1) /|U|}, j \leq d
$$

and

$$
\sum_{i=1}^{t} X_{i}^{p-1}
$$

with

$$
t=\frac{d(p-1)}{|U|}+p
$$

the proof follows as before.

To see that $f_{U}(p, d)>p+d-1$ when $U$ is the group of quadratic residues and $d \leq(p-1) / 2$, consider the sequence $(0, \cdots, 0)$ repeated $p-1$ times, along with the $d$ elements $(1,0, \cdots, 0),(0,1, \cdots, 0), \cdots(0,0, \cdots, 1)$ and the $d$ elements $(-t, 0, \cdots, 0),(0,-t, \cdots, 0), \cdots(0,0, \cdots,-t)$ where $\mathbf{Z}_{p}^{*}=U \sqcup t U$. Clearly, it has no zero-sum of length $p$ with weights from $U$.

\section{Proof of (iii).}

Clearly, a sequence of length $D_{U}(p, 1)-1$ which has no zero-sum subsequence with weights in $U$ can be augmented with the sequence $(0, \cdots, 0)$ repeated $p-1$ times and the combined sequence cannot contain a zero-sum subsequence of length $p$. This proves the inequality $f_{U}(p, 1) \geq p-1+D_{U}(p, 1)$. Since the inequality $f_{U}(p, 1) \leq p+\frac{p-1}{|U|}$ was proved in (ii) above, one has the equality $f_{U}(p, 1) \leq p+\frac{p-1}{|U|}$ whenever one has $D_{U}(p, 1) \leq 1+\frac{p-1}{|U|}$.

\section{Remarks.}

Similar to what we observed in the case of $D_{U}(p, d)$, one has that equality may not hold in (ii) of the above proposition, in general. For instance $f_{U}(7,2)<13$ when $U$ is the subgroup of cubic residues. 
The other method which is often useful in deducing results on zero-sums, is to use the Cauchy-Davenport theorem which states :

If $A_{1}, \cdots, A_{h}$ are non-empty subsets of $\mathbf{Z}_{p}$, then

$$
\left|A_{1}+\cdots+A_{h}\right| \geq \min \left(p, \sum_{i=1}^{h}\left|A_{i}\right|-h+1\right) .
$$

Using this, one has, for $a_{1}, \cdots, a_{r} \in \mathbf{Z}_{p}^{*}$ and for a subset $A$ of $\mathbf{Z}_{p}$, that

$$
\left|a_{1} A+\cdots+a_{r} A\right| \geq \min (p, r|A|-r+1) .
$$

In [4], it was shown that when $n=p_{1} p_{2} \cdots p_{k}$ is square-free and coprime to 6 , then $f_{U}(n, 1)=n+2 k$. For prime $n$, this is a consequence of (ii) of proposition 3 above - in fact, an inductive argument can be used to deduce this result for general square-free $n$. Now, we prove a generalization of proposition 11 from [4] where subgroups more general than the subgroup of squares are treated; this is the following :

\section{Proposition 4.}

(i) Let $n=p_{1} p_{2} \cdots p_{k}$ be odd and, square-free and, let $U_{i} \leq \mathbf{Z}_{p_{i}}^{*}$ be nontrivial subgroups. Consider the subgroup $U \leq \mathbf{Z}_{n}^{*}$ mapping isomorphically onto $U_{1} \times$ $U_{2} \cdots \times U_{k}$ under the isomorphism $\mathbf{Z}_{n}^{*} \rightarrow \mathbf{Z}_{p_{1}}^{*} \times \cdots \times \mathbf{Z}_{p_{k}}^{*}$ given by the Chinese remainder theorem. Suppose $r \geq \max \left\{\frac{p_{i}-1}{\left|U_{i}\right|-1}: i \leq k\right\}$. Further, let $m \geq r k$ and let $a_{1}, \cdots, a_{m+(r-1) k}$ be a sequence in $\mathbf{Z}_{n}$. Then, there exists a subsequence $a_{i_{1}}, \cdots, a_{i_{m}}$ and elements $u_{1}, \cdots, u_{m} \in U$ such that $\sum_{j} u_{j} a_{i_{j}}=0 \in \mathbf{Z}_{n}$.

(ii) With $n, U$ as above, $f_{U}(n, 1) \leq n+k\left(\max _{i} b_{i}-1\right)$ where $b_{i}=\left\lceil\frac{p_{i}-1}{\left|U_{i}\right|-1}\right\rceil$.

\section{Proof.}

For the first part we proceed by induction on the number $k$ of prime factors of $n$.

If $k=1$, write $n=p$. If there are less than $r$ elements among $a_{1}, \cdots, a_{m+r-1}$ which are non-zero in $\mathbf{Z}_{p}$, then at least $m$ of them are zero. Hence, taking $m$ such $a_{i}$ 's and arbitrary units $u_{1}, \cdots, u_{m}$ the corresponding sum is zero. If, on the other hand, at least $r$ among the $a_{i}$ 's (say, $a_{1}, \cdots, a_{r}$ ) are in $\mathbf{Z}_{p}^{*}$, then the above observation based on the Cauchy-Davenport theorem shows that

$$
\left|a_{1} U+\cdots+a_{r} U\right| \geq \min (p, r|U|-r+1) .
$$

Now, $p \leq r|U|-r+1$ since it is given that $r \geq \frac{p-1}{|U|-1}$. 
Hence, $a_{1} U+\cdots+a_{r} U=\mathbf{Z}_{p}$. So, there are $u_{1}, \cdots, u_{r} \in U$ such that

$$
a_{1} u_{1}+\cdots+a_{r} u_{r}=-\left(a_{r+1}+\cdots+a_{m}\right) .
$$

Thus, the choice $u_{r+1}=\cdots=u_{m}=1$ gives $\sum_{i=1}^{m} u_{i} a_{i}=0$. Thus, the case $k=1$ follows.

Assume that $k \geq 2$ and that the result holds for smaller $k$.

Consider any sequence $a_{1}, \cdots, a_{m+(r-1) k}$ in $\mathbf{Z}_{n}$.

Suppose first that, for each $i \leq k$, at least $r$ among the $a_{i}$ 's are units modulo $p_{i}$. So, there is $t \leq r k \leq m$ such that among $a_{1}, \cdots, a_{t}$ there are at least $r$ units in $\mathbf{Z}_{p_{i}}$ for each $i \leq k$. Then, we have solutions of $\sum_{j=1}^{m} a_{j} u_{j}^{(i)} \equiv 0$ $\bmod p_{i}$ for $i=1, \cdots, k$ and $u_{j}^{(i)} \in U_{i}$ for each $j \leq m$. As $U$ is a subgroup of $\mathbf{Z}_{n}^{*}$ corresponding to the product $U_{1} \times U_{2} \cdots \times U_{k}$ by the Chinese remainder theorem, the group $U$ contains elements $u_{1}, \cdots, u_{m}$ such that $u_{j} \equiv u_{j}^{(i)} \bmod$ $p_{i}$ for $i=1, \cdots, k$. Therefore, $\sum_{j=1}^{m} u_{j} a_{j} \equiv 0 \bmod n$. We are done in this case.

Now, consider the case when the sequence of $a_{i}$ 's contain less than $r$ units mod $p_{i}$ for some $p_{i}$, say $p_{1}$. Removing them, we have a sequence of $m+(r-1) k-(r-1)=m+(r-1)(k-1)$ elements which are all $\equiv 0 \bmod p_{1}$. By induction hypothesis, the case $k-1$ implies that there is a subsequence $a_{s_{1}}, \cdots, a_{s_{m}}$ of this and elements $u_{1}^{(i)}, \cdots, u_{m}^{(i)} \in U_{i}$ for each $i \geq 2$ such that $\sum_{j=1}^{m} u_{j}^{(i)} a_{s_{j}} \equiv 0 \bmod p_{i}$ for every $i \geq 2$. Since $a_{s_{j}}$ 's are all $0 \bmod p_{1}$, it follows that $\sum_{j=1}^{m} a_{s_{j}} \equiv 0 \bmod p_{1}$. Choosing elements $u_{1}, \cdots, u_{m} \in U$ by the Chinese remainder theorem, we have $\sum_{j=1}^{m} u_{j} a_{s_{j}} \equiv 0 \bmod p_{i}$ for all $i \geq 1$. Thus, we have $\sum_{j=1}^{m} u_{j} a_{s_{j}} \equiv 0 \bmod n=p_{1} p_{2} \cdots p_{k}$. This completes the proof.

Taking $m=n$, (ii) follows from (i); one simply uses the observation that $n \geq k r$.

\section{Remarks.}

As has been remarked following Proposition 3, the upper bounds in the above proposition may not be tight. In fact, it can be checked that $f_{U}(13,1) \leq 15$ where $U$ is the subgroup consisting of cubes.

Finally, we partially generalize the result $f_{\{1,-1\}}(n, 2)=2 n-1$ proved in [1] for odd $n$. The following Proposition treats the problem for more general subgroups and for general $d$. We obtain only an upper bound.

\section{Proposition 5.}

Let $U$ be a subset of $\mathbb{Z}_{n}^{*}$ closed under multiplication. Suppose that for each 
prime $p$ dividing $n$, the set $\{u \bmod p: u \in U\}$ is a subgroup of $\mathbb{Z}_{p}^{*}$ of order at least d. Then,

$$
f_{U}(n, d) \leq 2 n-1 .
$$

Further, equality holds when $U=\{1,-1\}, d=2$ and $n$ is odd.

\section{Proof.}

This will be proved by induction on the number of prime factors of $n$ (counted with multiplicity). The prime case is covered by Proposition 3. Write $n=$ $\prod_{i=1}^{k} p_{i}^{l_{i}}$. Start with a sequence $\mathbf{a}_{\mathbf{1}}, \cdots, \mathbf{a}_{\mathbf{2 n}-\mathbf{1}}$ of length $2 n-1$ in $\mathbb{Z}_{n}^{d}$. Look at the subsequence $\mathbf{a}_{\mathbf{1}}, \cdots, \mathbf{a}_{\mathbf{2}} \mathbf{p}_{\mathbf{1}} \mathbf{- 1}$. Since $\{u \bmod p: u \in U\}$ is a subgroup of $\mathbb{Z}_{p}^{*}$ of order at least $d$, Proposition 3(ii) gives a $p_{1}$-subsequence, say $\mathbf{a}_{\mathbf{1}}, \cdots, \mathbf{a}_{\mathbf{p}_{\mathbf{1}}}$ and elements $u_{1}^{\prime}, \cdots, u_{p_{1}}^{\prime} \in\left\{u \bmod p_{1}: u \in U\right\}$ such that $\sum_{i=1}^{p_{1}} \mathbf{a}_{\mathbf{i}} u_{i}^{\prime}=0$ in $\left(\mathbb{Z}_{p_{1}}\right)^{d}$. This means that $\sum_{i=1}^{p_{1}} \mathbf{a}_{\mathbf{i}} u_{i}=p_{1} \mathbf{b}_{\mathbf{1}}$ for some tuple $\mathbf{b}_{\mathbf{1}}$. Keeping away this $p_{1}$-subsequence and working with the rest, we get another $p_{1}$-sequence. We may, in this manner choose $2 m-1$ such subsequences (where $n=m p_{1}$ ) and corresponding elements in $U$ such that

$$
\sum_{i=j p_{1}+1}^{(j+1) p_{1}} \mathbf{a}_{\mathbf{i}} u_{i}=p \mathbf{b}_{\mathbf{j}+\mathbf{1}} \quad \forall 0 \leq j \leq 2 m-2 .
$$

Then, by induction hypothesis, one has elements $v_{1}, \cdots, v_{m}$ in $U$ and a $m$ subsequence, say, $\mathbf{b}_{\mathbf{1}}, \cdots, \mathbf{b}_{\mathbf{m}}$ so that $\sum_{j=1}^{m} \mathbf{b}_{\mathbf{j}} v_{j}=m \mathbf{b}_{\mathbf{0}}$ for some $d$-tuple $\mathbf{b}_{\mathbf{0}}$. Since $U$ is closed under multiplication modulo $n$, we will have then a pmsubsequence of the original sequence and elements of $U$ such that the sum is $0 \bmod n$. The equality $f_{U}(n, 2)=2 n-1$ when $U=\{1,-1\}$ and $n$ is odd is clear from considering the sequence $(1,0)$ repeated $n-1$ times along with the sequence $(0,1)$ repeated $n-1$ times as well.

\section{Remarks.}

(i) There are many examples of $U$ satisfying the hypothesis of the above theorem apart from $U=\{1,-1\}$ which was considered in [1]. For instance, the whole of $\mathbb{Z}_{n}^{*}$ is one such. More generally, if $n=p_{1} p_{2} \cdots p_{r}$ is square-free, then for any subgroups $U_{i} \leq \mathbb{Z}_{p_{i}}^{*}$, the Chinese remainder theorem gives us a subgroup $U$ of $\mathbb{Z}_{n}^{*}$ isomorphic to the product of the $U_{i}$ 's.

(ii) Using the above method, one can also prove the following result about the Davenport constant. If $n=\prod_{i=1}^{k} p_{i}^{l_{i}}$ is the prime factorization of $n$, then

$$
D_{U(n, r)}(n, d) \leq \prod_{i=1}^{k} D_{U\left(p_{i}, r\right)}\left(p_{i}, d\right)^{l_{i}} \leq \prod_{i=1}^{k}\left\{\frac{d\left(p_{i}-1\right)}{\left(p_{i}-1, r\right)}+1\right\}^{l_{i}} .
$$


Here, we have denoted by $\mathbb{Z}_{n}^{*}$, the group of units of $\mathbb{Z} / n \mathbb{Z}$ and, for $r \geq 1$, write $U(n, r)=\left\{u^{r}: u \in \mathbb{Z}_{n}^{*}\right\}$. Note that $\left|U\left(p_{i}, r\right)\right|=\frac{p_{i}-1}{\left(p_{i}-1, r\right)}$.

\section{Acknowledgement.}

We are indebted to the referee for pointing out a small gap in the earlier version of proposition 1 (iii).

\section{References}

[1] S.D.Adhikari, R.Balasubramanian, F.Pappalardi \& P.Rath - Some zerosum constants with weights, Proc. Indian Acad.Sci.. (Math.Sci.) 118, No. 2, 183-188 (2008).

[2] S.D. Adhikari and Y. G. Chen, Davenport constant with weights and some related questions II., J. Combin. Theory Ser. A 115, No. 1, 178184 (2008).

[3] S. D. Adhikari, Y. G. Chen, J. B. Friedlander, S. V. Konyagin and F. Pappalardi, Contributions to zero-sum problems, Discrete Math. 306, 1-10 (2006).

[4] S.D.Adhikari, C.David \& J.J.Urroz, Generalizations of some zero-sum theorems, Integers, Electronic Journal of Combinatorial Number Theory 8, A 52, (2008).

[5] Sukumar Das Adhikari and Purusottam Rath, Davenport constant with weights and some related questions, Integers 6, A30, (2006).

[6] N. Alon and M. Dubiner, A lattice point problem and additive number theory, Combinatorica 15, 301-309 (1995).

[7] D. J. Grynkiewicz, L. E. Marchan and O. Ordaz, A weighted generalization of two theorems of Gao, Priprint.

[8] H. Harborth, Ein Extremalproblem für Gitterpunkte, J. Reine Angew. Math. 262/263, 356-360 (1973).

[9] A. Kemnitz, On a lattice point problem, Ars Combin., 16b, 151-160 (1983). 
[10] J. E. Olson, A combinatorial problem in finite abelian groups, I, J. Number Theory, 1, 8-10 (1969).

[11] J. E. Olson, A combinatorial problem in finite abelian groups, II, J. Number Theory, 1, 195-199 (1969).

[12] Christian Reiher, On Kemnitz's conjecture concerning lattice-points in the plane, Ramanujan J., 13, No. 1-3, 333-337 (2007).

[13] L. Rónyai, On a conjecture of Kemnitz, Combinatorica, 20 (4), 569-573 (2000).

[14] Pingzhi Yuan and Xiangneng Zeng, Davenport constant with weights, Preprint. 